\title{
ARTICLE Alcohol withdrawal drives depressive behaviors by activating neurons in the rostromedial tegmental nucleus
}

\author{
Rao Fu', Wanhong Zuo ${ }^{1}$, Nimisha Shiwalkar ${ }^{1}$, Qinghua Mei ${ }^{1}$, Qing Fan ${ }^{1}$, Xuejun Chen ${ }^{1}$, Jing Li ${ }^{1}$, Alex Bekker ${ }^{1}$ and Jiang-Hong Ye ${ }^{1}$
}

\begin{abstract}
Rostromedial tegmental nucleus (RMTg) GABA neurons exert a primary inhibitory drive onto midbrain dopamine neurons and are excited by a variety of aversive stimuli. There is, however, little evidence that the RMTg-ventral tegmental area (VTA)-nucleus accumbens shell (Acb) circuit plays a role in the aversive consequences of alcohol withdrawal. This study was performed in adult male Long-Evans rats at 48-h withdrawal from chronic alcohol drinking in the intermittent schedule. These rats displayed clear anhedonia and depression-like behaviors, as measured with the sucrose preference, and forced swimming tests. These aberrant behaviors were accompanied by a substantial increase in cFos expression in the VTA-projecting RMTg neurons, identified by a combination of immunohistochemistry and retrograde-tracing techniques. Pharmacological or chemogenetic inhibition of RMTg neurons mitigated the anhedonia and depression-like behaviors. Ex vivo electrophysiological data showed that chemogenetic inactivation of RMTg neurons reduced GABA release and accelerated spontaneous firings of VTA dopamine neurons. Finally, using a functional hemispheric disconnection procedure, we demonstrated that inhibition of unilateral RMTg, when combined with activation of D1 and D2 dopamine receptors in the contralateral (but not ipsilateral) Acb, mitigated the anhedonia and depressionlike behaviors in alcohol-withdrawal rats. These data show that the integrity in the RMTg-VTA-Acb pathway in a single hemisphere is sufficient to elicit depression-like behavior during ethanol-withdrawal. Overall, the present results reveal that the RMTg-VTA-Acb pathway plays a crucial role in the depression-like behavior in animals undergoing alcohol withdrawal, further advocating the RMTg as a potential therapeutic target for alcoholism.
\end{abstract}

Neuropsychopharmacology (2019) 44:1464-1475; https://doi.org/10.1038/s41386-019-0378-8

\section{INTRODUCTION}

Alcohol-use disorder (AUD) is a chronic relapsing brain disease. Like most abused drugs, alcohol is both rewarding and aversive. Alcohol's rewarding properties are mainly mediated by its ability to increase the activity of dopamine neurons in the ventral tegmental area (VTA) and the release of dopamine in the projection targets, including the nucleus accumbens (Acb) [1-3]. Alcohol withdrawal from alcohol-dependent animals is often associated with a profound decremented mesolimbic dopamine function [4-6]. Clinical and preclinical studies suggest that diminished dopamine neurotransmission could be a common mechanism in both depression [7-10] and alcohol withdrawal [4-6].

The rostromedial tegmental nucleus (RMTg) comprising a cluster of gamma-aminobutyric acid (GABA)-ergic neurons, receives strong and direct excitatory inputs from the lateral habenula (LHb) [11-14] and exerts a major inhibitory drive onto midbrain dopamine neurons $[13,15]$. These dopamine neurons, in turn, project heavily to the Acb, thus forming a well-recognized pathway in addiction behaviors [16].

RMTg neurons encode negative reward signals $[12,17]$ and are excited by aversive events [12, 17-19]. As an essential inhibitory afferent to midbrain dopamine neurons, the RMTg is also implicated in drugs of abuse [18, 20-23], including alcohol [24-26]. The LHb has also emerged as a critical brain region in the pathophysiology of depression [27-29]. As a primary relay between the LHb and the VTA, the RMTg may function as an essential link in the neurocircuitry of alcohol withdrawal and relapse drinking, and therefore a potential target for therapeutic options for neuropsychiatric disorders [30].

To test the hypotheses that alcohol withdrawal excites RMTg neurons, leading to decreased dopamine neuronal activity, we measured neuronal firing in the RMTg/VTA, and the inhibitory postsynaptic currents (IPSCs) in dopamine neurons using electrophysiological recording combined with the inhibitory DREADD (Designed Receptor Exclusively Activated by Designed Drug) technique [31]. We also tested the hypothesis that RMTg hyperfunction accounts for depressive symptoms by reducing VTA-dopamine transmission onto the Acb of alcohol-withdrawal rats, and that pharmacological and/ or chemogenetic inactivation of the RMTg would attenuate the depressive-like behaviors in alcohol-withdrawal rats [32].

\section{MATERIALS AND METHODS}

Animals

Experiments were performed on a total of 214 adult male LongEvans rats $(250-300 \mathrm{~g}$ at the start of the studies, from Harlan Lab,

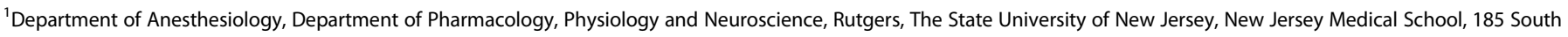
Orange Ave, Newark, NJ 07103, USA

Correspondence: Jiang-Hong Ye (ye@njms.rutgers.edu)

These authors contributed equally: Rao Fu, Wanhong Zuo

Received: 11 July 2018 Revised: 18 March 2019 Accepted: 24 March 2019

Published online: 31 March 2019 
Table 1. Summary of the groups for animal behavioral test

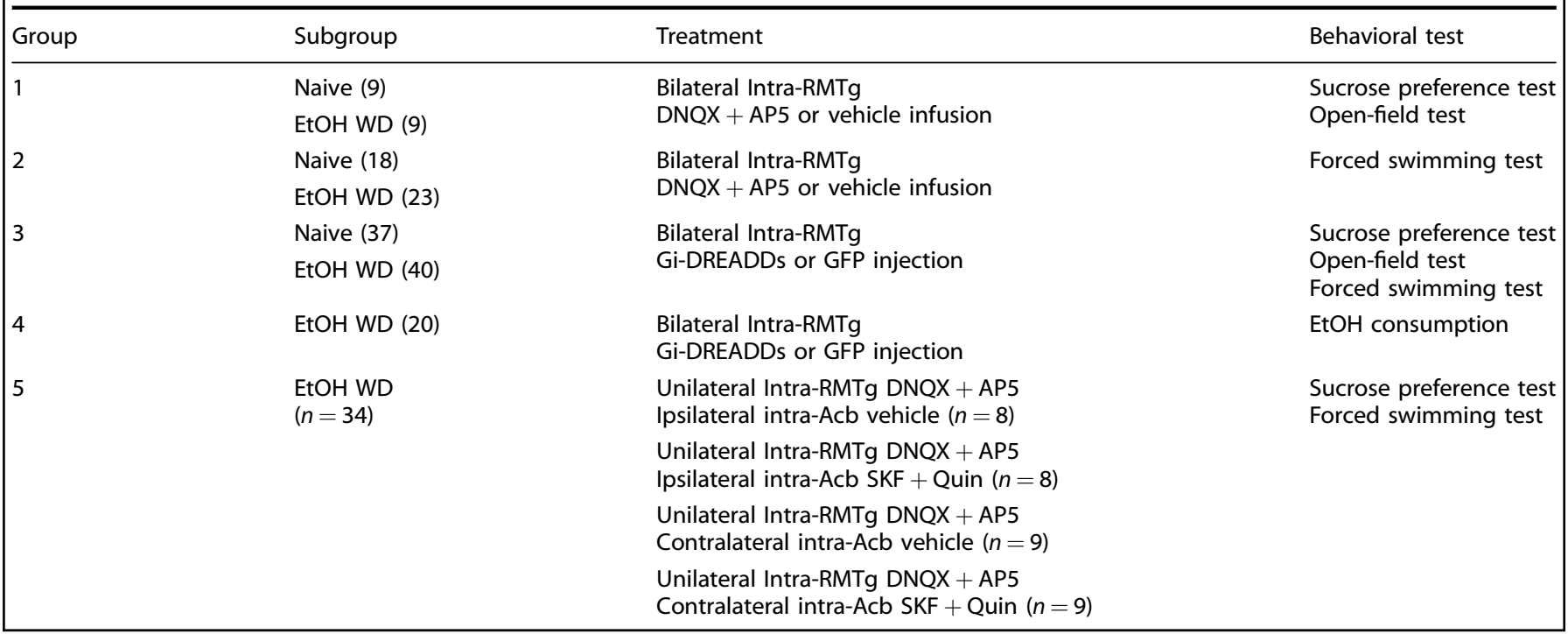

NY). The numbers of 190 rats used in behaviroal experiment are listed in Table 1. The rats were individually housed and kept on a 12/12 h light/dark cycle (lights off at 11:00 a.m.) in ventilated Plexiglas cages in a climate-controlled room $\left(20-22^{\circ} \mathrm{C}\right)$, with food and water ad libitum unless indicated otherwise. Animals were acclimatized to the housing conditions and handling for at least 7 days before the start of the experiments. All experimental procedures were performed by the guidelines of the National Institutes of Health for the Care and Use of Laboratory Animals and approved by the Institutional Animal Care and Use Committees of Rutgers, The State University of New Jersey, Newark, New Jersey.

We examined the effect of pharmacological inhibition of the RMTg on the sucrose preference test (SPT) and the open-field test (OFT) in the same group of rats in a counterbalanced manner. To minimize the potential influence of prior behavioral test on the next one, we kept the minimal interval between tests for 7 days. We performed the forced swimming test (FST) on a separate cohort of rats. In the chemogenetic inactivation of the RMTg experiments, each rat received three tests in the following sequence: SPT, OFT, and FST. In the functional cerebral disconnection experiments, SPT was performed before FST in the same group of rats.

Intermittent access to $20 \%$ ethanol in 2-bottle free-choice (IA2BC) drinking procedure

We measured ethanol intake in the IA2BC drinking paradigm, as described in refs. [33-35]; see Supplementary Materials and Methods for details.

Stereotactic surgery and microinjection procedure

Stereotactic surgery and histological verification were performed, as described in ref. $[25,36,37]$. For microinfusion chemicals to the Acb, a unilateral guide cannula (26 gauge; Plastics One, Wallingford, CT) was placed $1 \mathrm{~mm}$ above the Acb (in $\mathrm{mm}$ : A/P: 1.4-1.6; $\mathrm{M} / \mathrm{L}: \pm 1.4 ; \mathrm{D} / \mathrm{V}:-6.9$ from skull surface). For microinfusion chemicals to the RMTg, a unilateral guide cannula (26 gauge; Plastics One) was placed $1 \mathrm{~mm}$ above the RMTg (in $\mathrm{mm}: \mathrm{A} / \mathrm{P}:-6.8$; $\mathrm{M} / \mathrm{L}: \pm 0.45 ; \mathrm{D} / \mathrm{V}$ : -6.9 from skull surface); see Supplementary Materials and Methods.

Brain slice preparation and electrophysiology

Brain slices were prepared, as described in ref. [38]; see Supplementary Materials and Methods.
Immunohistochemistry and Immunofluorescence

The procedures involving brain fixation, immunofluorescence, and all antibodies used in this study were described in the "Supplementary Materials and Methods" section.

Chemicals and application

We purchased chemicals and common salts from Sigma-Aldrich Corporation (St. Louis, MO), ethanol from Pharmco Products (Brookfield, CT). In total, 6,7-di-nitroquinoxaline-2,3-dione (DNQX) and 2-amino-5-phosphonopentanoic acid (AP5) were from Tocris. Clozapine-N-oxide (CNO) was obtained from NIDA Drug supply program (NIH, Bethesda, MD). The detail for drugs preparation was described in the "Supplementary Materials and Methods" section.

Measurement of anhedonia and depressive-like behaviors SPT and FST were conducted in alcohol-naive rats, and rats at 48 $\mathrm{h}$ withdrawal from the last ethanol-drinking session (EtOH-WD), see Supplementary Materials and Methods.

RMTg/VTA-Acb shell functional disconnection studies

The functional disconnection procedure was described in refs. [39, 40]. Briefly, rats at 48 - $h$ withdrawal from the last ethanol-drinking session, before SPT and FST, received a unilateral intra-RMTg infusion of DNQX + AP5 (5 and $10 \mathrm{pmol} / 100 \mathrm{nl} / \mathrm{side}$ ) combined with ipsilateral or contralateral intra-Acb shell infusion of SKF38393+ quinpirole (SKF + Quin, $500 \mathrm{pmol} / 500 \mathrm{nl} / \mathrm{side})$ or vehicle $(n=8-9$ / group). At the end of behavioral tests, cannula placements were assessed by Nissl staining using light microscopy.

Statistical analysis

All data were expressed as a mean \pm SEM (standard error of the mean). To calculate the percent change in SIPSCs and firing for a given cell, recordings during the first control period (baseline) were averaged and normalized to $100 \%$. Statistical significance was assessed using the paired or unpaired $t$ test, one-or two-way ANOVA or two-way repeated measures ANOVA, followed by a Bonferroni's post hoc test. Statistical significance was declared at $p<0.05$.

\section{RESULTS}

Alcohol withdrawal enhances cFos expression in VTA-projecting RMTg neurons

Ethanol consumption of rats in the $\mathrm{IA} 2 \mathrm{BC}$ paradigm was significantly escalated, from $2.56 \pm 0.22 \mathrm{~g} / \mathrm{kg} / 24 \mathrm{~h}$ in the first 

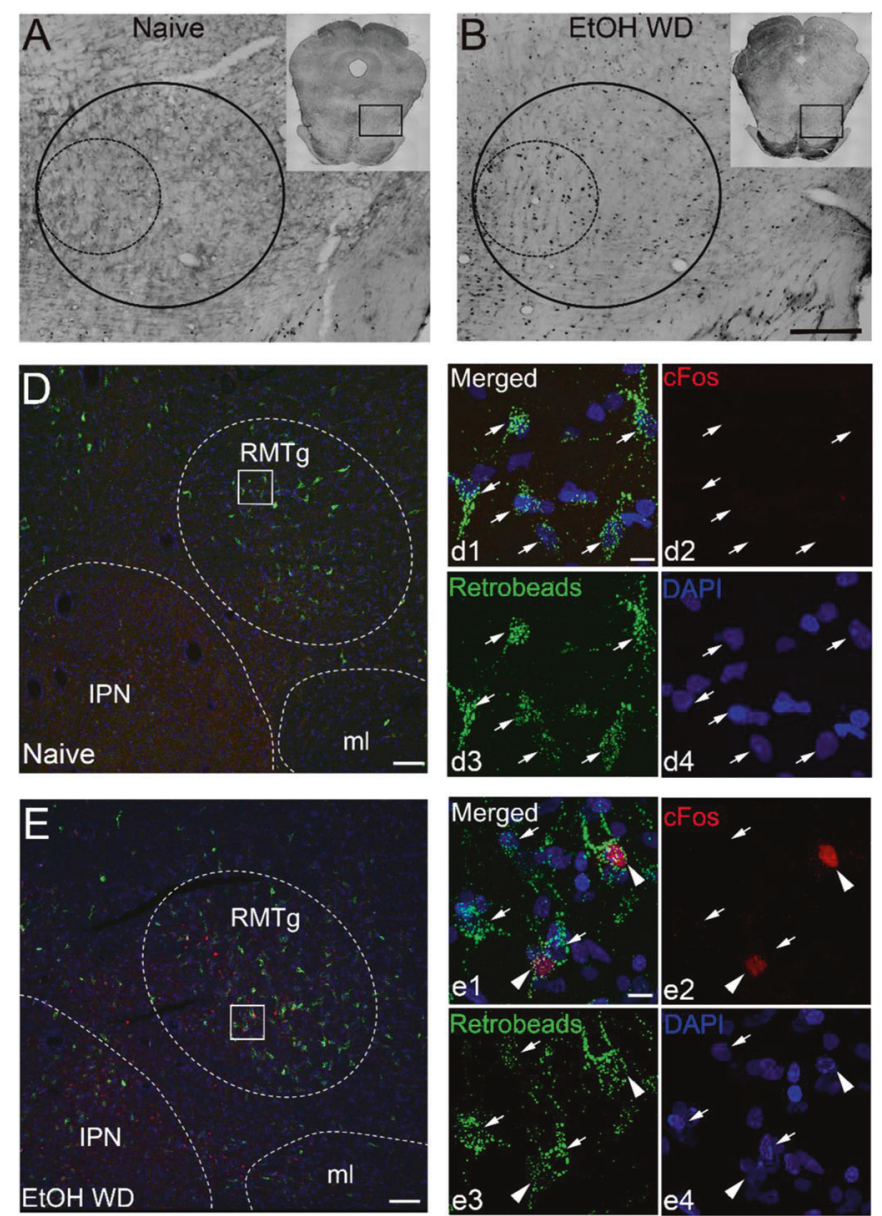

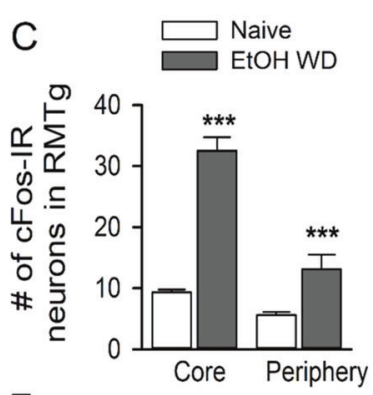

$\mathrm{F}$
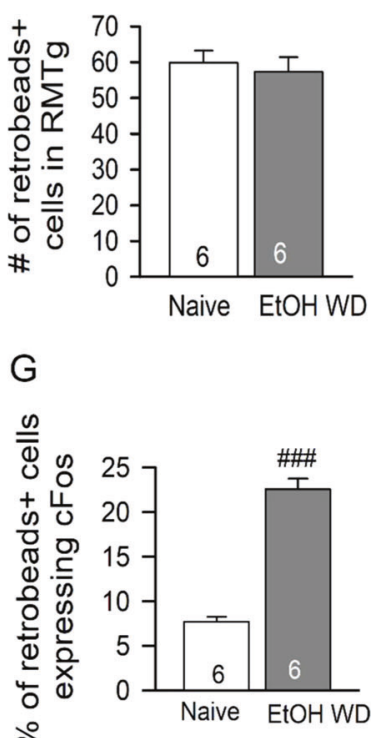

Fig. 1 c-Fos expression of the ventral tegmental area (VTA)- projecting rostromedial tegmental nucleus (RMTg) neurons is increased in alcohol-withdrawal rats. $\mathbf{a}, \mathbf{b}$ are representative images showing c-Fos-immunoreactive (IR) neurons in RMTg core (outlined in the dashed circle) and periphery (outlined in the solid circle) in rats of alcohol naive group (Naive) and 48-h withdrawal from chronic (10 weeks) intermittent alcohol consumption group (EtOH-WD). The boxed areas in the insets are enlarged in the corresponding panels. Scale bar $=$ $200 \mu \mathrm{m}$. c summary of RMTg cFos-IR cell counting shows cFos-IR RMTg neurons were substantially increased in EtOH-WD rats as compared with Naive counterparts (for alcohol treatment effect, $F_{1,23}=86.064, p<0.001$, post hoc test, ${ }^{* * *} p<0.001$ vs. naive, $n=6 /$ groups), and the increase was greater in the core than the peripheral (for location: $F_{1,23}=29.072, p<0.001$, post hoc test, ${ }^{\wedge \wedge \wedge} p<0.001$ vs. core, $n=6 /$ groups), revealed by two-way ANOVA followed by Bonferroni $t$ test. d, e are representative images showing the VTA projecting RMTg neurons (retrobeads labeled, green) expressed cFos (red). Magnified pictures demonstrate the merged RMTg cells (d1,e1) in the areas outlined by the white soild box in $\mathrm{d}$ and e. White arrows indicate the neurons expressing cFos $(\mathrm{d} 2, \mathrm{e} 2)$ and are retrogradely labeled (d3,e3), as well as counterstained by DAPI (d4, e4) of naive and EtOH-WD rats. f, $\mathbf{g}$ Alcohol withdrawal did not significantly alter the number of retrogradely labeled cells in the RMTg (f), but enhances the proportion of retrobeads labeled cells that expressing cFos (g). The animal numbers used are shown inside the bars. ${ }^{\# \#} p<0.001$ vs. Naive, two-tailed unpaired $t$ test. All data were presented in mean \pm S.E.M. Scale bar $=100 \mu \mathrm{m}$ (d, e) or $10 \mu \mathrm{m}(\mathrm{d} 1, \mathrm{e} 1)$

session to $6.23 \pm 0.34 \mathrm{~g} / \mathrm{kg} / 24 \mathrm{~h}$ at the 24 th session (8 weeks), which maintained the same levels after that, consistent with previous reports $[33,34,41]$. There were substantially more c-Fos IR neurons in the RMTg of rats at 48 - $h$ withdrawal from chronic alcohol (EtOH-WD) than that in the alcohol-naive control (Naive) rats (Fig. 1a-c). c-Fos expression was increased in both the core and the periphery of the RMTg [12] and was higher in the core than the periphery $(32.6 \pm 2.2$ in core vs. $13.1 \pm 2.4$ in the periphery, $t=2.898, p=0.016, n=6$ rats/group, unpaired $t$ test).

To find VTA-projecting RMTg neurons that were activated during alcohol withdrawal, we combined the retrograde-tracing technique with cFos-IR. The number of VTA-projecting RMTg neurons in the EtOH-WD rats was not much different from that of naive control $(59.9 \pm 3.4$ in Naive, $57.3 \pm 4.1$ in EtOH-WD, $t=0.476$, $p=0.644, n=6$ rats/group, two-tailed unpaired Student's $t$ test, Fig. 1f). However, the proportion of retrograde-labeled neurons expressing cFos was significantly greater in EtOH-WD rats than in the naive counterparts $(7.7 \pm 0.6$ in Naive, $22.6 \pm 1.2$ in EtOH-WD, $t=11.363, p<0.001, n=6$ rats/group, two-tailed unpaired Student's $t$ test, Fig. 1g). This result suggests that the connection between the RMTg and the VTA has been strengthened after chronic alcohol administration and withdrawal.

Chemogenetic inactivation or pharmacological inhibition of the RMTg alleviates anhedonia- and depressive-like behaviors in alcohol withdrawal rats

Consistent with recent results [32], in rats at 48 -h withdrawal from chronic alcohol drinking, there were clear enhancement in the immobility in the forced swimming test (FST, Supplementary Figure $1 \mathrm{~A}-\mathrm{C}$ ) and reduction in the consumption of $1 \%$ sucrose solution in the sucrose preference test (SPT, Supplementary Figure 1D, E). To examine the potential role of the RMTg in these behaviors, we injected $\mathrm{Gi}$-coupled designer receptor exclusively activated by the designer drug (DREADD; hM4Di) or the control vector (GFP) into the bilateral RMTg of 84 rats (Fig. 2a). Histological verification confirmed 77 rats with the expression of viral vectors 
A

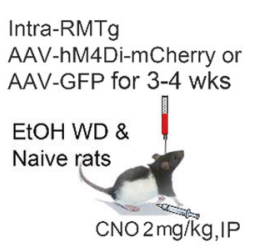

C

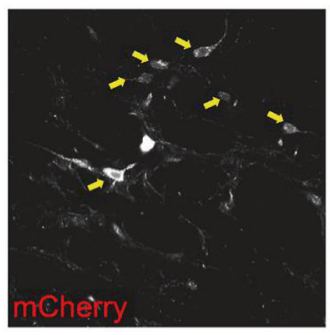

E

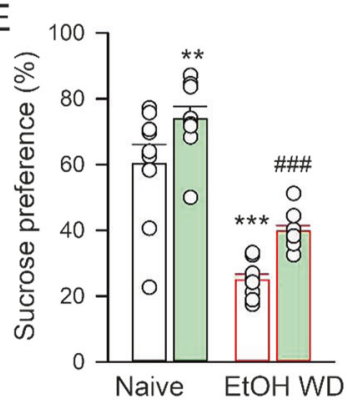

$\mathrm{H}$

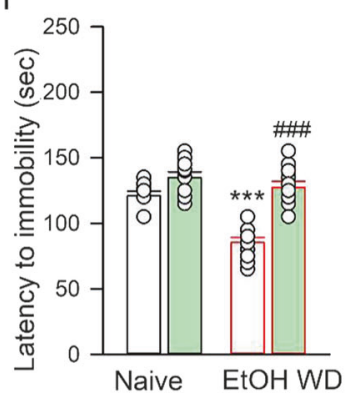

B
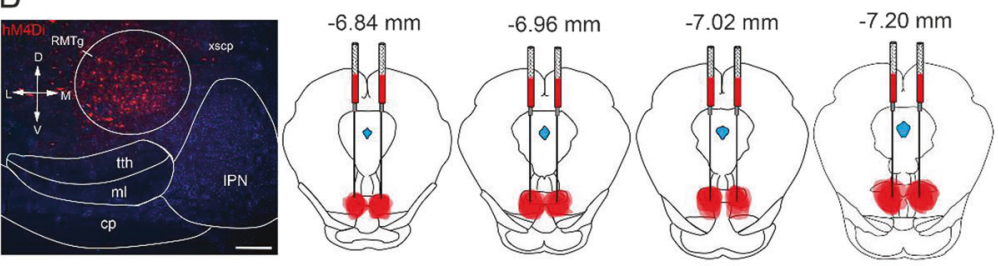

D
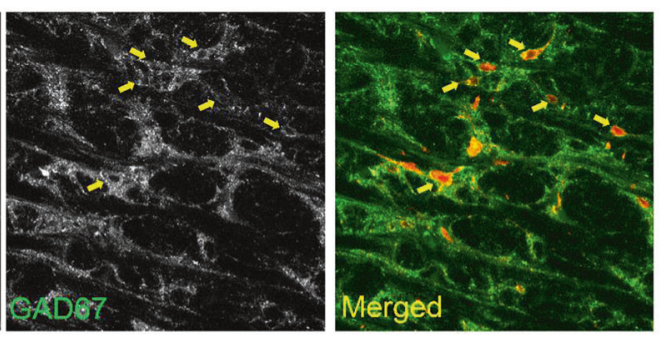

Total RMTg hM4Di

mCherry+ cells

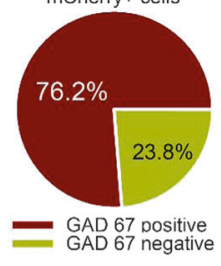

hM4Di
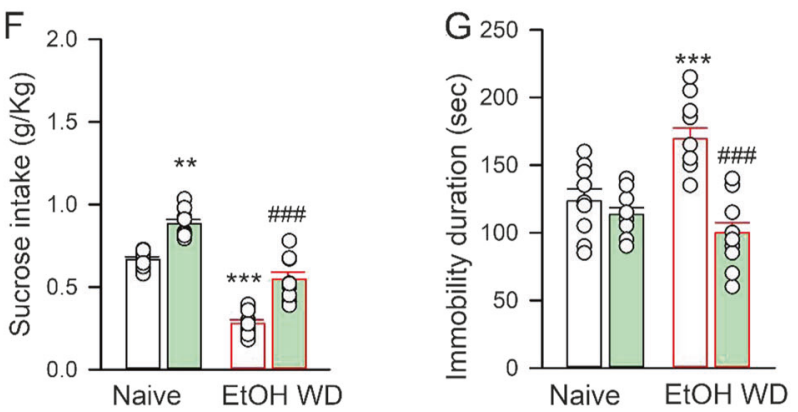

I

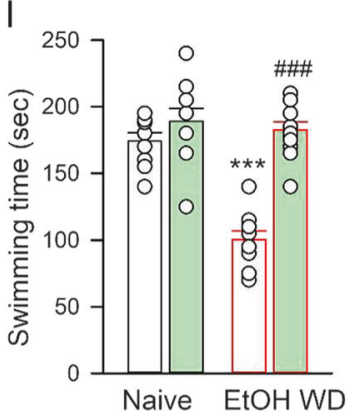

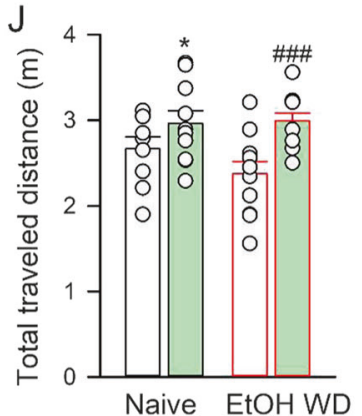

Fig. 2 Chemogenetic inactivation of the rostromedial tegmental nucleus (RMTg) neurons alleviates anhedonia- and depression-like behaviors in alcohol-withdrawal rats. a The schematic view of a rat injected with hM4Di (Gi)-DREADDs viral vector. CNO clozapine-N-oxide, IP intraperitoneal. b Left panel, hM4Di-mcherry expression in the RMTg after viral vector injection; right panel, virus expression sites are plotted on four coronal sections from a rat brain atlas [71]. All hM4Di viral sites $(n=39)$ are presented at the level of their maximal extents. Approximate anteroposterior distance to bregma is indicated on each drawing. IPN interpeduncular nucleus, ml medial lemniscus, tth trigeminothalamic tract. Scale bar $=200 \mu \mathrm{m}$. c Representative photomicrographs of the RMTg neurons with mCherry (yellow arrow), GAD67 (yellow arrow) immunoreactivity, and merge (yellow arrow) image from a rat injected with AAV-hM4Di-mCherry into the RMTg. Scale bar = $50 \mu \mathrm{m}$. d Pie chart summarizes the proportion of GAD67 + and GAD67- neuronal populations of mCherry + neurons in the RMTg (RMTg $\mathrm{mCherry}+$ cell counting $=47.2 \pm 7, n=6$ rats). The preference for $(\mathbf{e})$ and consumption of $(\mathbf{f}) 1 \%$ sucrose by the naive and EtOH-WD rats $(n=9$ per group) during the RMTg chemogenetic inactivation. Two-way repeat measurement ANOVA group effect $F_{1,35}=41.078, p<0.001$; virus effect $F_{1,35}=56.366, p<0.001$; group $\times$ virus interaction $F_{1,35}=0.0926, p=0.769$ for sucrose preference, and group effect $F_{135}=77.804, p<$ 0.001 ; virus effect $F_{1,35}=127.551, p<0.001$, group $\times$ virus interaction, $F_{1,35}=1.325, p=0.283$ for sucrose intake. The forced swimming and locomotor activity tests in EtOH-WD and naive rats $(n=9-11$ per group) during RMTg chemogenetic inactivation. Two-way ANOVA virus effect $F_{1,40}=28.914, P<0.001$; group $\times$ virus interaction $F_{1,40}=16.097, P<0.001$ for immobile duration (g); virus effect $F_{1,40}=43.601, P<0.001$, group $\times$ virus interaction, $F_{1,40}=10.973, P=0.002$ for latency to immobility (h); virus effect $F_{1,40}=42.874, P<0.001$, group $\times$ virus interaction, $F_{1,40}=20.703, P<0.001$ for swimming time (i); virus effect $F_{1,40}=11.722_{* * *} P=0.002$, group $\times$ virus interaction, $F_{1,40}=1.457, P=0.235$ for total

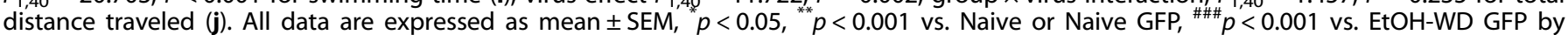
Bonferroni's post hoc test 
within the RMTg (Fig. 2b). The data of the seven rats that missed virus expression in the RMTg were excluded from analysis. Cell counting based on confocal immunofluorescence images showed that $76.2 \%$ of virally transfected neurons (mCherry labeled) in the RMTg expressed glutamic acid decarboxylase 67 (GAD67), a marker of GABAergic neurons, implying recruitment of GABAergic neurons in the RMTg (Fig. 2c, d).

We evaluated the RMTg's role in the SPT in 36 rats. The preference and consumption of either $1 \%$ sucrose or water during a 4-h session were measured at $48 \mathrm{~h}$ after the last ethanol-drinking session. CNO (2 mg/kg, I.P.), an agonist of Gi DREADD, administrated $30 \mathrm{~min}$ before SPT significantly increased sucrose preference and consumption in both $\mathrm{EtOH}-\mathrm{WD}$ and naive rats infected with AAV-hM4Di, but not in rats infected with AAV-GFP (Fig. 2e, f).

We evaluated the role of the RMTg in the FST in other 41 rats, which were divided into four groups: $\mathrm{EtOH}-\mathrm{WD}$ and naive groups, injected with either AAV-hM4Di $(n=22)$ or AAV-GFP $(n=19)$ into the RMTg. CNO (2 mg/kg, I.P.) shortened the immobility time in EtOH-WD rats infected with AAV-hM4Di, but not with AAV-GFP (Fig. 2f-h). However, compared with the vehicle, CNO treatment did not change the immobility of naive rats infected with either AAV-hM4Di or GFP. Interestingly, CNO significantly increased the locomotor activity in both EtOH-WD and naive rats infected with AAV-hM4Di, but not GFP. These data suggest that chemogenetic inactivation of the RMTg effectively rescued the anhedonia- and depression-like behaviors associated with alcohol withdrawal.

Next, we examined the effect of chemogenetic inactivation of the RMTg on alcohol consumption in a group of rats $(n=20)$ that had been drinking ethanol in the IA2BC paradigm for 6 weeks. These rats later received intra-RMTg AAV-hM4Di or AAV-GFP injection and re-accessed to ethanol after 7-days recovery from surgery. The viral injection procedure did not significantly alter the alcohol consumption on all injected rats $(6.17 \pm 0.43$ vs. $6.41 \pm$ $0.46 \mathrm{~g} / \mathrm{kg} / 24 \mathrm{~h}, \mathrm{t}(18)=1.284, p=0.218$ by paired $t$ test). Also, no significant difference was found between the hM4Di and GFP groups $\left(6.27 \pm 0.33\right.$ vs. $6.56 \pm 0.32 \mathrm{~g} / \mathrm{kg} / 24 \mathrm{~h}, t_{(18)}=0.3, p=0.769$ by $t$ test). At $4-5$ weeks after virus injection, we examined the effect of $\mathrm{CNO}$ in a counterbalanced way. A histology study confirmed 16 rats with RMTg virus expression (Supplementary Figure 2). Administration of CNO (2 mg/kg, I.P.), but not the vehicle significantly increased the intake of and the preference for ethanol, as well as the total fluid intake in rats infected with RMTg hM4Di, but not those infected with GFP (Supplementary Figure 2).

Also, we examined the effect of pharmacological blockade of glutamate transmission on the RMTg on the SPT and FST. We infused the cocktail of DNQX $(50 \mu \mathrm{M})$ and AP5 $(100 \mu \mathrm{M})$, the selective AMPA and NMDA receptor antagonists into the RMTg of EtOH-WD and naive rats 10 min before the SPT and FST, at 48-h withdrawal from the last drinking session. Histological examination verified 59 rats had bilateral injector tip sites within the RMTg (Fig. 3a). Compared with aCSF, intra-RMTg infusion of DNQX plus AP5 made both EtOH-WD and naive rats consume significantly more sucrose, as well as enhanced their preference to sucrose solution over water (Fig. 3b, c). Intra-RMTg DNQX and AP5 also reduced the immobile duration, prolonged the latency to first immobility and the swimming time in EtOH-WD rats (Fig. 3i-m), and increased locomotor activity in both EtOH-WD and naive rats.

CNO inhibits spontaneous firings of RMTg neurons infected with AAV-hM4Di

We examined the effect of $\mathrm{CNO}$ on the spontaneous firings of RMTg neurons in slices of 12 rats. The basal firing rates of RMTg neurons in rats infected with GFP were like those infected with hM4Di (Fig. 4a-d). A two-way RM-ANOVA revealed a significant group $\times$ treatment interaction $\left(F_{1,20}=5.7, p=0.027\right.$, Fig. $\left.4 d\right)$. A post hoc test showed that bath application of CNO (1 $\mu \mathrm{M}, 10 \mathrm{~min})$ reversibly decreased the firing rate of 8 out of 12 RMTg neurons expressing hM4Di ( $p=0.001$; Fig. $4 c, d$ ), but had no effect on RMTg neurons expressing GFP ( $p=0.79$; Fig. $4 b$, d). Moreover, CNO $(1-5 \mu \mathrm{M})$ dose-dependently and significantly inhibited the firings of RMTg neurons expressing hM4Di $\left(F_{3,27}=9.6, p<0.001\right.$; Fig. $4 \mathrm{e})$, compared with the vehicle control (0.05\% DMSO).

Chemogenetic inhibition of GABA transmission from the RMTg to VTA-DA neurons increases the activity of VTA-DA neurons

Whole-cell voltage-clamp recordings were made from putative dopamine (DA) neurons in the VTA, and spontaneous GABA $A_{A}$ IPSCS (sIPSCs) were examined (Fig. 4a) in brain slices from 12 rats. Bath application of CNO $(1-5 \mu \mathrm{M})$ was used to activate hM4Di expressed on the terminals of RMTg neurons that projected to the VTA. The neurons recorded in the VTA meet conventional electrophysiological criteria of dopamine neurons (Fig. 5b1, c1). No significant difference was found about the frequency and amplitude of basal sIPSCs recorded from putative DA neurons in rats whose RMTg was infected with hM4Di or GFP (Fig. 5b, c). Bath application of $1 \mu \mathrm{M}$ CNO did not affect either the frequency or the amplitude of sIPSCs in VTA-DA neurons (Fig. 5b, d1, e1) infected with GFP, but substantially reduced the frequency of sIPSCs and right-shifted the cumulative distribution of inter-event intervals on most DA neurons infected with hM4Di (7 out of 10 cells). A two-way RM ANOVA for group $\times$ treatment further confirmed that in RMTg-hM4Di rats there was a significant interaction effect on SIPSC frequency $\left(F_{1,19}=4.6, p=0.044\right.$; post hoc $p=0.005$ pre-CNO vs. CNO; Fig. $5 d 1)$, but not on sIPSC amplitude $\left(F_{1,19}=0.7, p=0.43\right.$; Fig. $\left.5 \mathrm{e} 1\right)$. Thus, $\mathrm{CNO}$ selectively and dose-dependently reduced the presynaptic inhibitory synaptic transmission to the DA neurons in the VTA of rats whose RMTg was infected with hM4Di (frequency: $F_{3,30}=10.2, p<0.001$; amplitude: $F_{3,40}=1.1, p=0.38$; Figs. $5 \mathrm{~d} 2$, e2).

To determine whether inactivating GABA transmission in the RMTg will alter the output of VTA-DA neurons, we examined the effect of hM4Di activation on the spontaneous firings of VTA-DA neurons. Bath perfusion of $1 \mu \mathrm{M}$ CNO slightly but significantly increased $(14.5 \pm 5.2 \%$ increase, $n=11$ cells from five rats) the firing rate of VTA-DA neurons in slices from rats infected with hM4Di in the RMTg, but not the firing rate of VTA-DA neurons in rats infected with GFP in the RMTg $(0.25 \pm 0.77 \%$ increase, $n=$ 11 cells from four rats) ( $t=2.47, p=0.014$, hM4Di vs. GFP). These results show that activation of hM4Di on RMTg terminals in the VTA results in increased dopamine neuron activity.

The anti-depressant effect of RMTg inhibition is mediated by dopamine transmission in the nucleus accumbens

Mesolimbic dopamine transmission has been implicated in depression [42], and dopamine neurons projecting to the nucleus accumbens $(A c b)$ shell receive inhibitory input from the RMTg [43, 44]. We hypothesized that the activation of the VTA-projecting RMTg GABAergic neurons might underlie the decrease in the extracellular dopamine levels in the Acb $[6,45]$, representing the neural basis of the aversive consequences observed after abrupt cessation of chronic alcohol intake. To better assess the role of RMTg-VTA-Acb circuit in anhedonia and depressive behaviors during alcohol withdrawal, we performed a functional hemispheric disconnection experiment (see the Materials and Methods section). A unilateral cannula was implanted into the RMTg or Acb shell of a group of adult male Long-Evans rats that had been drinking ethanol in the IA2BC paradigm for 10 weeks. One week after the surgery, these rats resumed drinking in the same IA2BC schedule for an additional week. SPT and FST were performed during the 48 - $\mathrm{h}$ withdrawal period from the last drinking session. Ten to $15 \mathrm{~min}$ prior to SPT and FST, all rats universally received unilateral RMTg inhibition (by DNQX + AP5); they were then randomly assigned to four subgroups: the 1st subgroup received the vehicle and the 2nd subgroup a cocktail of SKF-38393 and quinpirole (SKF + Quin; respectively, selective dopamine D1 and 

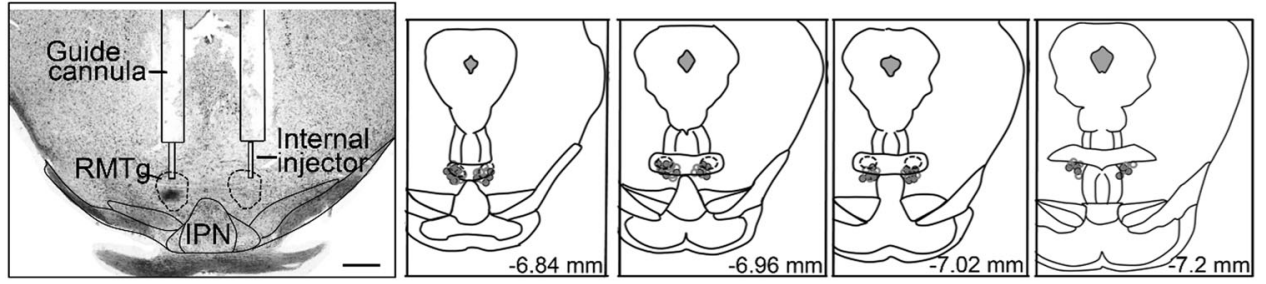

Vehicle שIIA DNQX+AP5
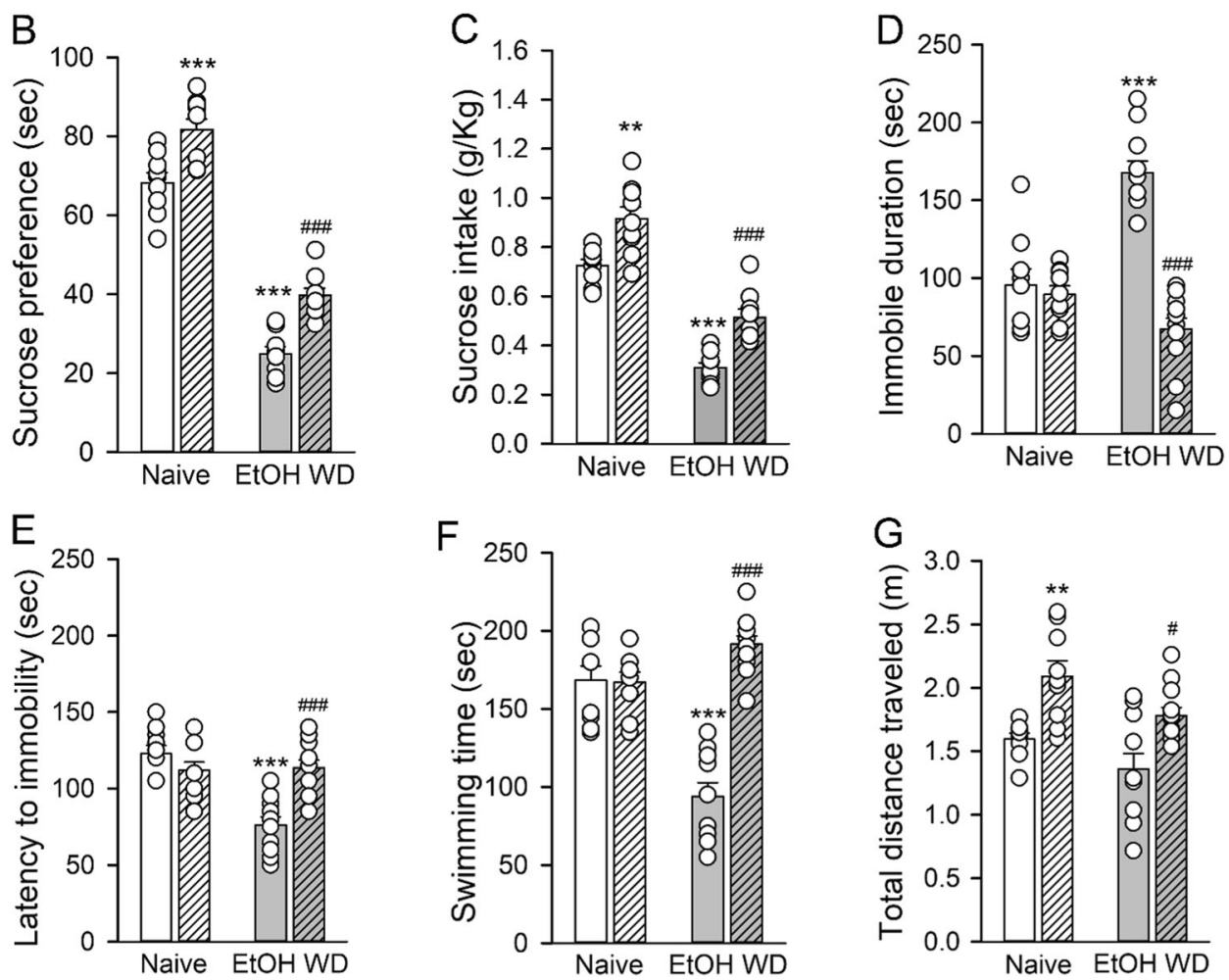

Fig. 3 Blockade of glutamate transmission on the rostromedial tegmental nucleus (RMTg) neurons improves anhedonia and depression-like behavior in alcohol-withdrawal rats. a Left panel, representative microphotograph of Nissl staining showing bilateral intra-RMTg guide cannula placement. Right panel, schematic drawings of coronal sections of the rat brain showing the tips of injector placements from individual EtOH-WD ( $n=32$, red blank circles) and naive rats $(n=27$, gray solid circles) with accurate bilateral cannula placements in the RMTg (adapted from [71]). IPN interpeduncular nucleus. Scale bar $=1 \mathrm{~mm}$. The preference for and intake of $1 \%$ sucrose solution of EtOH-WD and naive rats $(n=9$ per group) after intra-RMTg infusion DNQX $(50 \mu \mathrm{M})+$ AP5 $(100 \mu \mathrm{M})$. Two-way repeat measurement ANOVA group effect $F_{1,35}=224.327, p<0.001$; treatment effect $F_{1,35}=405.791, p<0.001$; group $\times$ treatment interaction $F_{1,35}=0.487, p=0.505$ for sucrose preference (b), and group effect $F_{1,35}=106.573, p<0.001$; treatment effect $F_{1,35}=405.791, p<0.001$, group $\times$ treatment interaction, $F_{1,35}=$ $0.255, p=0.627$ for sucrose intake (c). The swimming behavior and locomotor activity test of EtOH-WD and naive rats ( $n=9-12$ per group) after intra-RMTg infusion of DNQX + AP5. Two-way ANOVA group effect $F_{1,41}=11.413, p<0.001$; treatment effect $F_{1,41}=49.475, p<0.001$; group $\times$ treatment interaction: $F_{1,38}=39.625, p<0.001$ for immobile duration (d); group effect $F_{1,41}=11.282, p=0.002$; treatment effect $F_{1,41}=41.768, p<0.001$; group $\times$ treatment interaction: $F_{1,38}=44.115, p<0.001$ for latency to immobility (e); group effect $F_{1,41}=25.634, p=$ 0.002 ; treatment effect $F_{1,41}=4.892, p=0.003$; group $\times$ treatment interaction: $F_{1,38}=28.12, p<0.001$ for swimming time (f). Group effect $F_{1,38}=31.652, p=0.002$ on total distance traveled $(\mathbf{g})$. All data are expressed as mean $\pm \mathrm{SEM},{ }^{* *} p<0.01,{ }^{* * * *} p<0.001$ vs. Naive or Naive vehicle, ${ }^{\#} p<0.05,{ }^{\# \#} p<0.001$ vs. EtOH-WD vehicle by Bonferroni's post hoc test

D2 agonists), in their ipsilateral Acb shell. The 3rd subgroup received the vehicle and the 4th subgroup SKF38393 + quinpirole in their contralateral Acb shell. Histological verification revealed that the cannula tips of 34 rats (from 40) were within the RMTg and the Acb shell, and the data of these rats were used for the analysis (Fig. 6a, b).

In the SPT, rats in the 1st, 2nd, and 3rd subgroups significantly reduced sucrose preference and consumption compared with the control alcohol-naive group (Fig. 6c, d). Whereas, rats in the 4th subgroup significantly increased sucrose preference and consumption compared with the other (1st, $2 \mathrm{nd}$, and $3 \mathrm{rd}$ ) subgroups.
This result suggests that the integrity of RMTg-VTA-Acb circuit in one hemisphere is sufficient to elicit anhedonia in alcoholwithdrawal rats. A similar result was seen in the FST. Rats in the 1st, 2nd, and 3rd subgroups significantly increased immobile duration and decreased swimming time, as compared with the control alcohol-naive group. Rats in the 4th subgroup had significantly shorter immobility time, and longer swimming time than the other (1st, 2nd, and 3rd) subgroups (Fig. 6e, f). This result suggests that the integrity of RMTg-VTA-Acb circuit in one hemisphere is a pre-requisite for depressive behavior in alcoholwithdrawal rats. These data thus show that the RMTg-VTA-NAc 
A

Day 0

Day 21

Injected GFP/hM4Di into RMTg

B1

GFP-control

Pre-CNO

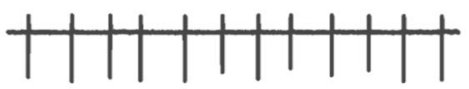

$1 \mu \mathrm{M} \mathrm{CNO}$
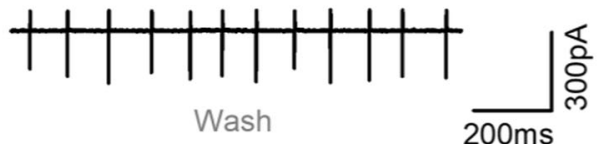

Wash

$200 \mathrm{~ms}$

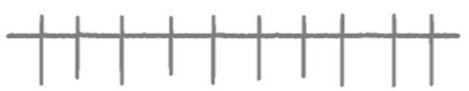

Recorded on RMTg neurons

C1 hM4Di-DREADD

Pre-CNO
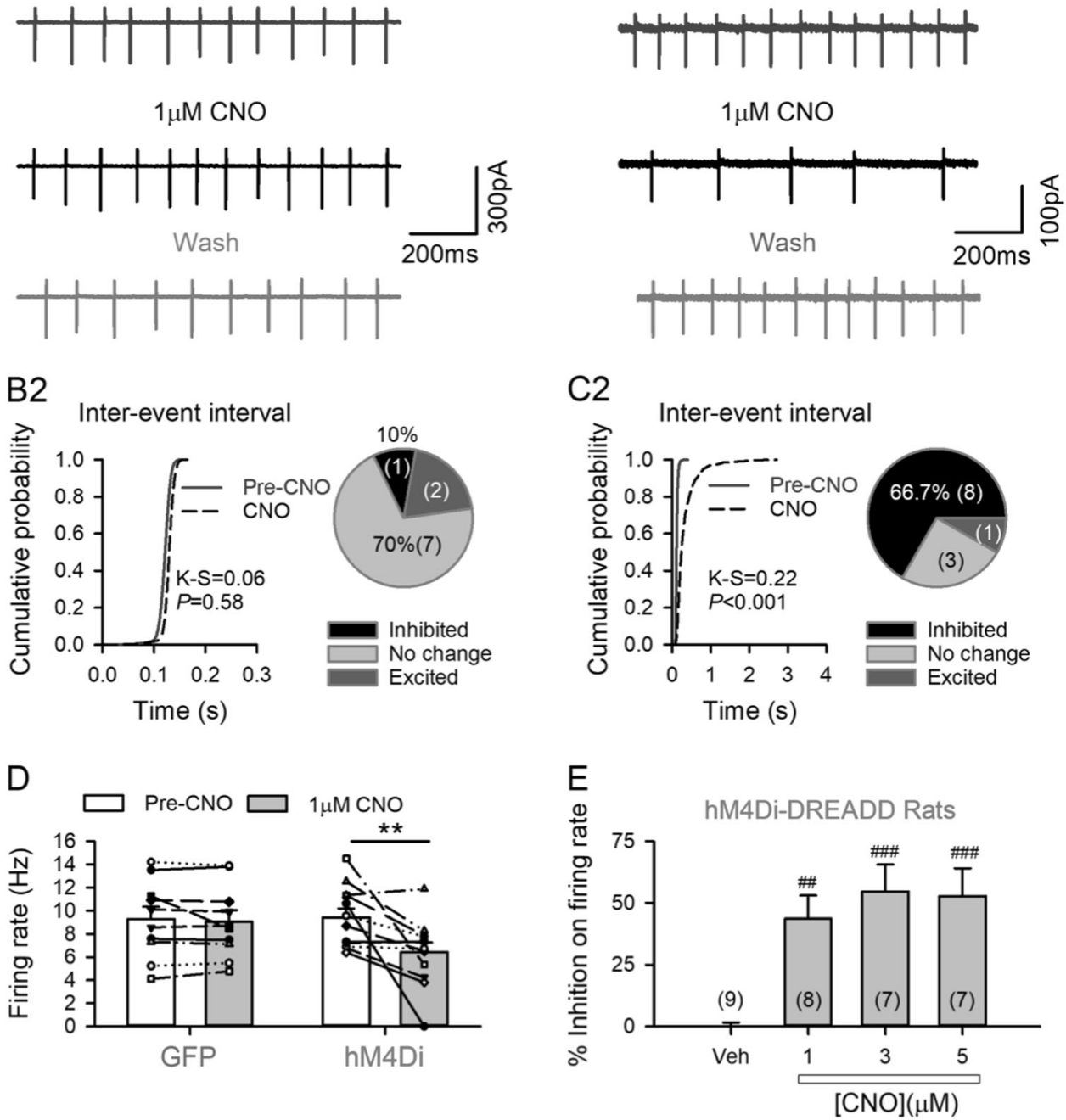

Fig. 4 Chemogenetic inactivation of the rostromedial tegmental nucleus (RMTg) neurons. a The experimental scheme. AAV-hM4Di-mCherry or AAV-GFP were bilaterally infused into the RMTg. Cell-attached recording from the RMTg was performed 21 days later. Representative traces of spontaneous firings of RMTg neurons infected with GFP (b1) or hM4Di (c1) before (upper), during (middle), and after (bottom) bath application of $1 \mu \mathrm{M}$ CNO. Cumulative probability plots for the firing inter-event interval in GFP (b2) and hM4Di (c2) groups. CNO caused a substantial suppression of firing in 8/11 RMTg neurons infected with hM4Di, but not with GFP. d Mean \pm SEM and individual values of $1 \mu \mathrm{M}$ CNO effects on RMTg firing rate in GFP and hM4Di infected rats. ${ }^{* *} p<0.01$, two-way RM ANOVA followed by Bonferroni's post hoc test. e Summary of the vehicle (Veh, $0.05 \%$ DMSO) and CNO $(1,3,5 \mu \mathrm{M})$ induced $\%$ inhibitions of firing rate in hM4Di infected rats. ${ }^{\# \#} p<0.01,{ }^{\# \#} p<$ 0.001 one-way ANOVA

circuit plays a crucial role in the dysphoric state during alcohol withdrawal.

\section{DISCUSSION}

Rats withdrawn from chronic alcohol administration showed anhedonia- and depressive-like behaviors as measured by the sucrose preference and forced swimming tests, which was accompanied by a substantial increase in cFos expression in VTA-projecting RMTg neurons. Unilateral RMTg inhibition, combined with the activation of D1 and D2 dopamine receptors in the contralateral but not ipsilateral Acb shell, mitigated these aberrant behaviors, suggesting the integrity in the RMTg-VTA-Acb pathway in one hemisphere is sufficient for these behaviors. Inactivation of RMTg neurons reduced IPSCs in dopamine neurons, but accelerated the firing of dopamine neurons. Although RMTg inhibition did not reduce alcohol consumption as we expected, these data strongly show that the RMTg-VTA-Acb circuit plays a critical role in the negative-affective disorders in alcoholwithdrawal animals.

We first confirmed the depressive-like behaviors in rats withdrawn from chronic IA2BC schedule [32, 46]. The symptoms of depression, including helplessness and anhedonia, were more often found in alcoholics during the period of alcohol withdrawal $[47,48]$. Depressive-like behavior has also been observed in rodents withdrawn from chronic intermittent exposure to 
A

Day 0

Injected GFP/hM4Di into RMTg
Day 35

Recorded on VTA DA neurons

B1

$$
\text { SynGFP-control }
$$

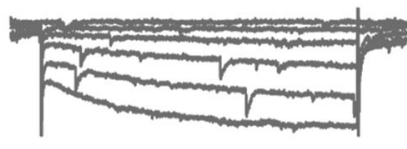

B2

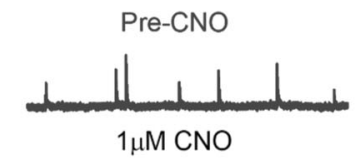

SIPSCs
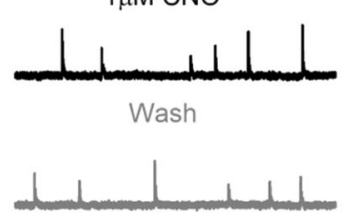

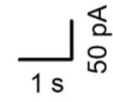

C1
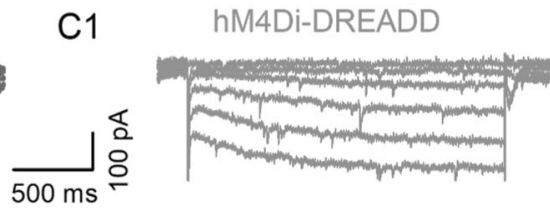

C2
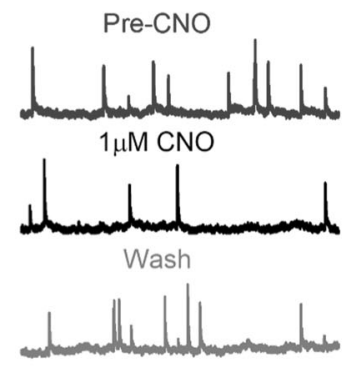
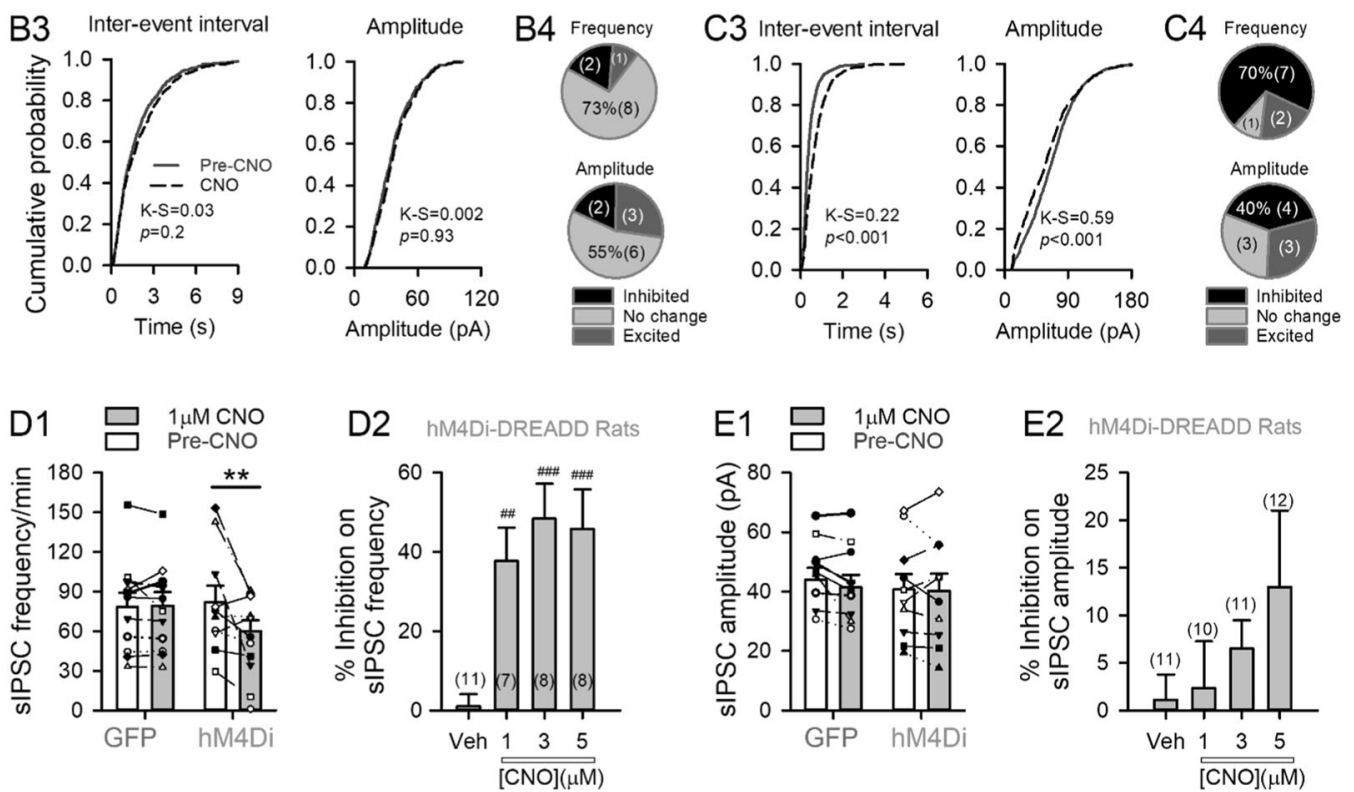

Fig. 5 Chemogenetic inactivation of the rostromedial tegmental nucleus (RMTg) terminals on VTA decreases GABA release onto VTAdopamine (DA) neurons. a The experimental scheme. AAV-hM4Di-mCherry or AAV-GFP were bilaterally infused into the RMTg. Whole-cell recording on putative DA neurons in the VTA was performed 35 days later. $\mathbf{b}, \mathbf{c}$ In the voltage-clamp mode, a series of hyperpolarizing steps (from a holding potential of -60 to $-110 \mathrm{mV}$ in $10-\mathrm{mV}$ increments) elicited prominent $I_{\mathrm{h}}$-like inward current relaxation in the putative DA neurons of GFP (b1), and hM4Di (c1) injected rats. Representative GABAergic sIPSC traces (b2, c2), and the cumulative probability plots of sIPSC amplitude and frequency $(b 3, c 3)$ in the absence and presence of $1 \mu \mathrm{M}$ CNO. c4, d4 Overall, $1 \mu \mathrm{M}$ CNO selectively reduced sIPSC frequency on most (70\%) VTA-DA neurons in hM4Di, but not in GFP infected rats. CNO did not have clear effects on sIPSC amplitude. d1, e1 Mean \pm SEM and individual values of CNO effects on sIPSC frequency and amplitude. ${ }^{* *} p<0.01$, two-way RM ANOVA. d2, e2 The vehicle (Veh) and CNO induced $\%$ inhibition on sIPSCs in hM4Di infected rats. ${ }^{\# \#} p<0.01,{ }^{\# \#} p<0.001$, one-way ANOVA

alcohol vapor $[49,50]$. Local infusion of DNQX and AP5 or chemogenetic inactivation of RMTg neurons alleviated these behaviors, confirming a critical role of the RMTg in depressive mood disorder [30].

We then showed that the depressive-like behavior was accompanied by the increased RMTg activity, as shown by the increased cFos expression. A similar phenomenon was reported in rats withdrawn from chronic intermittent exposure to ethanol vapor [51]. cFos expression in RMTg neurons increases after reward omission or the presence of negative stimuli [12], and during morphine withdrawal $[18,22,52]$. Alcohol dependence is characterized by the emergence of a negative-affective state [53]. We, therefore, infer that the negative-affective state during withdrawal from the IA2BC paradigm might activate the RMTg. The $\mathrm{LHb}$ provides principal excitatory inputs to RMTg-GABA neurons $[11-13,54]$. The $\mathrm{LHb}$ is hyperactive during alcohol withdrawal $[32,36,55]$. Therefore, the increased excitatory synaptic transmission from the LHb to RMTg might contribute to RMTg activation during alcohol withdrawal. Notably, most cFos-immunoreactive neurons in alcohol-withdrawal rats reside in the RMTg core, from where the neurons preferentially project to the VTA-DA neurons [12].

VTA-DA neurons are involved in reward coding and motivation processing. Acute alcohol exposure increases VTA-DA neuron firing and dopamine release in limbic structures [56-58]. Acute alcohol withdrawal decreases dopamine neuronal activity $[6,59]$, the number 
A
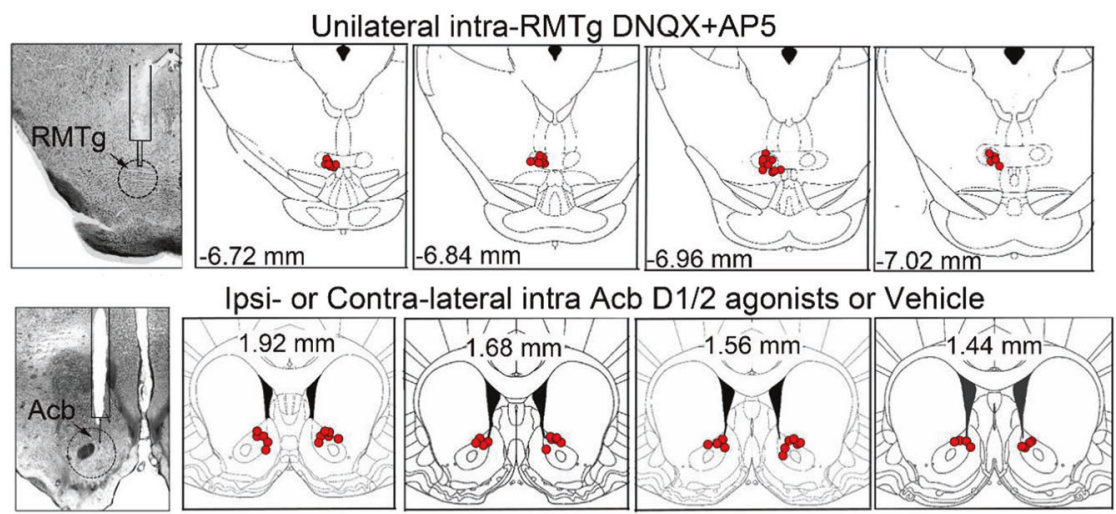

B
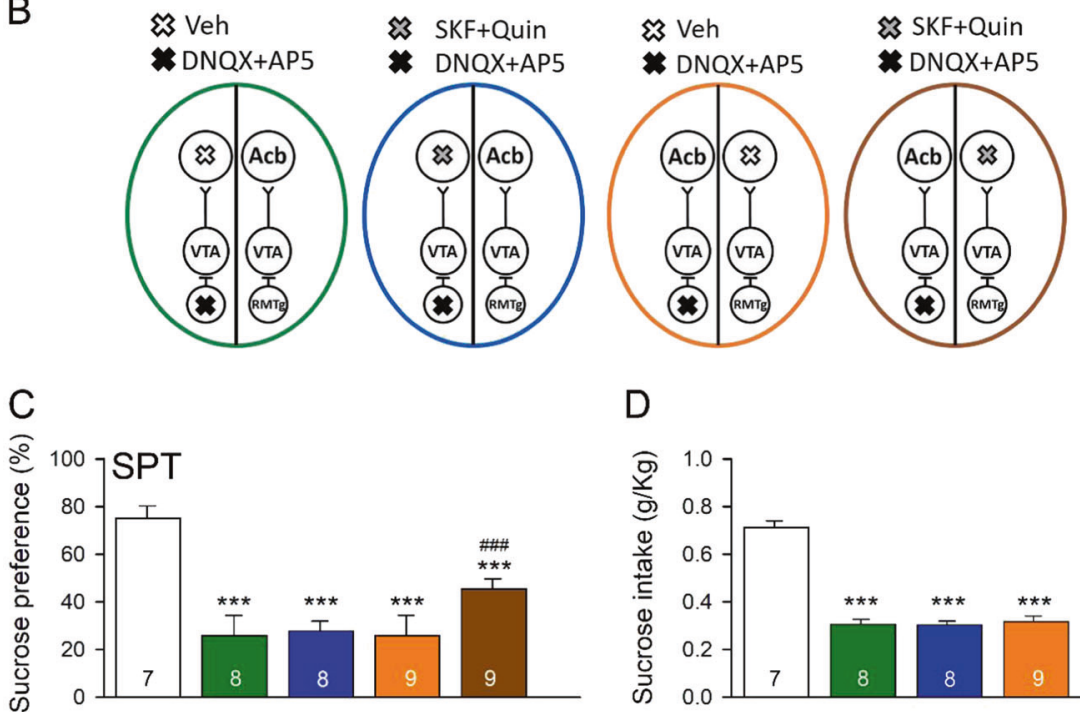

D

$\mathrm{E}$
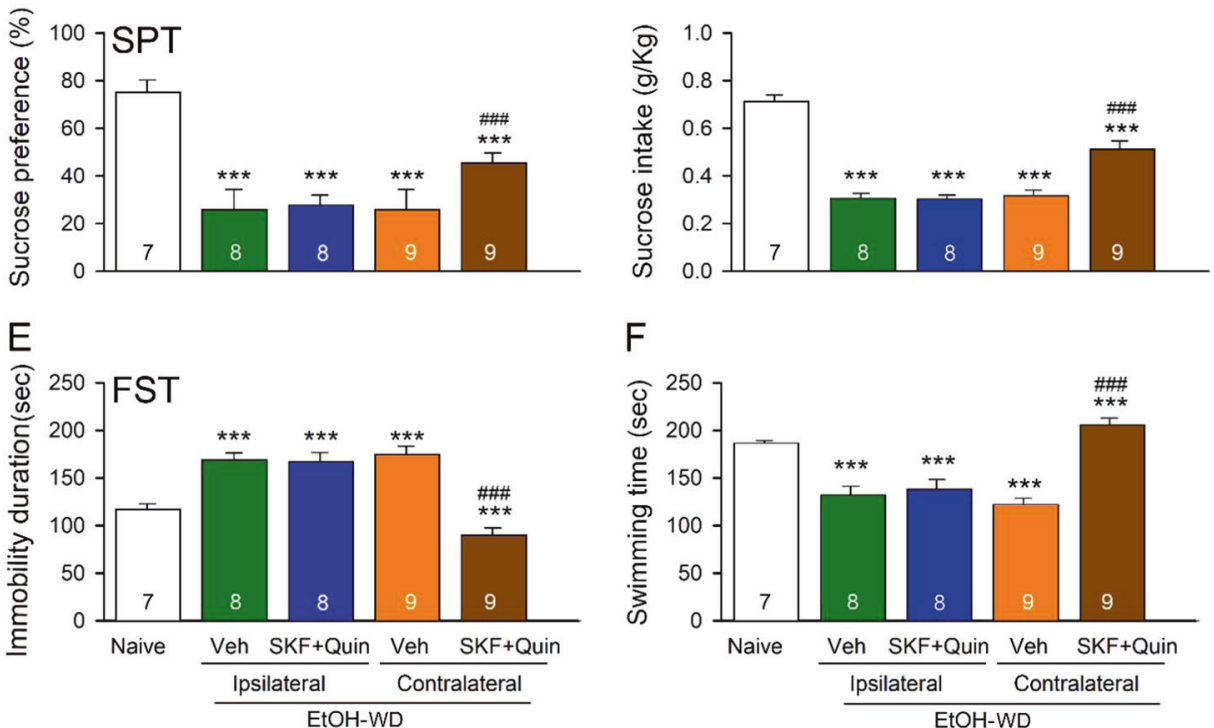

Fig. 6 Rostromedial tegmental nucleus (RMTg) regulation of depression-like behavior involves dopamine receptors in the nucleus accumbens (Acb) shell. a The experimental design for RMTg and Acb shell disconnection procedure in EtOH-WD rats. Left, representative microphotographs of Nissl staining showing injector placements in the RMTg (upper panel) and Acb shell (bottom panel). Right, diagrams of series of sections ordered from rostral (left) to caudal (right) showing infusion sites of the RMTg (upper panel) and Acb shell (bottom panel), as shown by the solid red dots. b Schematic representation of experimental groups with description of unilateral intra-RMTg injection of the glutamate receptor antagonists (DNQX + AP5) combined with intra-Acb infusion of vehicle (green) or D1 and D2 dopamine receptor agonizts (SKF + Quin, blue) in ipsilateral hemisphere, as well as with vehicle (orange) or SKF + Quin (brown) in contralateral hemisphere. c-f The data of sucrose preference test (SPT, sucrose preference, $F_{4,40}=43.213, P<0.001$; sucrose intake $F_{4,40}=45.429, P<0.001$ ) and forced swimming test (FST, immobile duration $F_{4,40}=19.715, P<0.001$; swimming time $F_{4,40}=23.078, P<0.001$ ) during pharmacological disconnection of RMTg and Acb shell circuits of EtOH-WD rats. All data were presented in mean \pm SEM. One-way ANOVA $p<0.001$ vs. control group, ${ }^{* \# \#} p<0.001$ vs. SKF + Quin or Veh in contralateral Acb, or Veh in contralateral Acb group by Bonferroni's post hoc test

of spontaneously active dopamine neurons [5], as well as dopamine levels in the Acb [6]. The majority of RMTg neurons ( 70-92\%) are GABAergic $[12,13]$; thus the RMTg is a significant source of inhibitory input to the midbrain dopamine neurons. Therefore, increased RMTg activity could account, at least in part, for the reduced activity of dopamine neurons during acute alcohol withdrawal.
Acute inhibition of VTA-DA neurons induces multiple distinct depressive-like behaviors [10]. The alleviation of depressive behavior may have resulted from a release of VTA-DA neurons from RMTg inhibition. This possibility is also supported by the evidence that transient removal of GABAergic inhibition increases phasic bursts of dopamine neurons $[12,60,61]$. Our results are 
also in accord with previous findings that RMTg ablation reduces anxiety-like behavior [12], and that injection of the anxiogenic drug $\beta$-carboline increases RMTg cFos expression [62, 63]. Thus, these studies suggest that RMTg activation may play a crucial role in keeping the response to aversive stimuli, including negative mood disorder.

Also, we used the SPT to investigate how RMTg regulates anhedonia-like behavior. The central dopamine dysfunction has been widely proposed as a common neurobiological correlate of the psychopathological expression of anhedonia [48, 64]. RMTg inactivation alleviated anhedonia in alcohol-withdrawal rats, as reflected by increased sucrose preference. Given that the RMTg tonically controls dopamine neuronal activity, these data suggest that RMTg regulation of anhedonia may involve the dopamine mesolimbic and mesocortical pathways. The purpose of the FST is to test the animals' internal motivational state ("escape or wait to

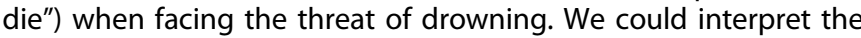
current observation as RMTg inactivation enhances motivation toward survival. Decreased sIPSCs in VTA-DA neurons accompanied the attenuated depressive-like behavior. The RMTg sends strong GABAergic inhibitory projections to the midbrain dopamine neurons, the dorsal raphe, and the pedunculopontine nucleus $[12,13,15,43,65]$, all of which are regions implicated in motivated behaviors. Therefore, RMTg inactivation may account for enhancing motivation and yield generalized behavioral activation via the regulation of several pathways. As for the general function of the RMTg, it has been described as particularly specialized for representing negative stimuli and for promoting inhibitory behavioral responses to these stimuli. Moreover, RMTg lesions selectively impair reactions involving reduction or withholding of motor activities (freezing and avoidance) and spared or enhanced active responses (startle, open-arm escape, and treading) [12]. However, it remains to be determined whether RMTg activity and mood disorders such as depressive behaviors have a causal relationship. e.g., whether RMTg excitation is enough to induce increased immobility during the FST or if depressive behavior per se could serve as an independent factor leading to RMTg excitation.

In the experiments in which DREADDs virus was injected into the RMTg, in addition to the RMTg, the interpeduncular nucleus (IPN) of some rats was also infected. Although we did not find a significant difference in the SPT and FST between rats with and without IPN infection, we could not exclude a potential role of IPN in regulating the depression-like behaviors, considering the critical role of the medial habenula-IPN circuitry in drug addiction, anxiety, and mood regulation [66]. Depressive behaviors correlate with dysfunction of mesolimbic dopamine neurons [42, 67]. Anatomically, the RMTg controls the Acb shell projecting VTA-DA neurons $[43,44]$. Using a pharmacological disconnection experiment, we linked together the potential regulatory role of the RMTg on the dopamine system and its related psychiatric disorders associated with alcoholism. The attenuation of depressive-like behavior and anhedonia seen in rats with unilateral RMTg inhibition combined with dopamine receptor activation in the contralateral Acb shell suggest that decreased dopamine transmission in the mesolimbic circuit, especially from VTA to Acb, may underlie the aversive states associated with alcohol withdrawal. Additionally, the anti-depressive activity of RMTg inhibition seems to be mediated through the activation of the dopamine receptors in the Acb shell. More importantly, the failure to rescue resulting from other combinations in the subgroups of alcohol-withdrawal rats suggest that the integrity of RMTg-VTA-Acb shell circuit in a single hemisphere might be a pre-requisite for expression of these symptoms during alcohol withdrawal. A limitation of our current study is that evidence connecting the RMTg, and the Acb is indirect; we saw depressivelike behaviors due to changes in the RMTg and the dopamine receptors in the Acb. Future studies to record the neuronal activity in the Acb while the RMTg is being manipulated will provide more direct evidence for the connection. Our data show that inhibition of Acb dopamine signaling underlies the depressive-like behaviors that were attenuated by RMTg inhibition. Notably, a previous study showed that phasic activation of VTA-DA neurons acutely rescues depression-like phenotype, which requires the functioning of dopamine receptors in the Acb [10].

Although we propose that RMTg hyperactivity may contribute to anhedonia and depressive behavior, which comprises withdrawal symptoms and might be a significant contributor to relapse $[68,69]$, we observed, contrary to expectation, that inactivation of RMTg neurons did not reduce alcohol consumption. Concerning alcohol consumption, the current data were in accord with our previous finding that pharmacological inhibition by muscimol or ablation of RMTg neurons enhances alcohol consumption [25, 37]. Considering the RMTg effectuates the aversive properties of cocaine [21], we interpret our drinking data to indicate that during RMTg inhibition as a result of a loss of balance between the rewarding and the aversive effects of alcohol, which influences voluntary drug intake [70]. Therefore, the reduction of RMTg response to alcohol's aversive properties may result in increased consumption.

\section{CONCLUSION}

We showed that depression-like behaviors during acute withdrawal from chronic alcohol consumption were accompanied by increased RMTg activity. Given that inhibition of the RMTg or activation of dopamine receptors in the Acb can alleviate the depression-like behaviors, it appears that the RMTg and the projection from the RMTg to the VTA-DA neurons are crucial sites in the depressive behaviors during alcohol withdrawal.

\section{FUNDING AND DISCLOSURE}

The authors report no financial interests or potential conflicts of interest. This work was funded by NIH grants AA021657 and AA022292, and a grant from New Jersey Health Foundation. Clozapine-n-oxide (CNO) was from NIDA Drug supply program (NIH, Bethesda, MD).

\section{ACKNOWLEDGEMENTS}

The authors appreciate Dr. S. Gupta for her reading over the paper.

\section{AUTHOR CONTRIBUTIONS}

$\mathrm{RF}$ and JY conceived and designed all behavioral experiments. RF and WZ performed stereotactic surgery. RF, QM, $\mathrm{QF}$, and $\mathrm{XC}$ performed behavioral tests. WZ performed electrophysiology. RF, NS, and JL performed immunofluorescence. RF, WZ, and JY performed the statistical analysis, prepared figures, and wrote the paper. All authors contributed to reviewing and editing the paper.

\section{ADDITIONAL INFORMATION}

Supplementary Information accompanies this paper at (https://doi.org/10.1038/ s41386-019-0378-8).

Publisher's note: Springer Nature remains neutral with regard to jurisdictional claims in published maps and institutional affiliations.

\section{REFERENCES}

1. Yim HJ, Gonzales RA. Ethanol-induced increases in dopamine extracellular concentration in rat nucleus accumbens are accounted for by increased release and not uptake inhibition. Alcohol. 2000;22:107-15.

2. Yoshimoto K, McBride WJ, Lumeng L, Li TK. Alcohol stimulates the release of dopamine and serotonin in the nucleus accumbens. Alcohol. 1992;9:17-22. 
3. Nicola SM, Malenka RC. Dopamine depresses excitatory and inhibitory synaptic transmission by distinct mechanisms in the nucleus accumbens. J Neurosci. 1997; 17:5697-710.

4. Rossetti ZL, Hmaidan Y, Gessa GL. Marked inhibition of mesolimbic dopamine release: a common feature of ethanol, morphine, cocaine and amphetamine abstinence in rats. Eur J Pharmacol. 1992;221:227-34.

5. Shen RY. Ethanol withdrawal reduces the number of spontaneously active ventral tegmental area dopamine neurons in conscious animals. J Pharmacol Exp Ther. 2003;307:566-72.

6. Diana M, Pistis M, Carboni S, Gessa GL, Rossetti ZL. Profound decrement of mesolimbic dopaminergic neuronal activity during ethanol withdrawal syndrome in rats: electrophysiological and biochemical evidence. Proc Natl Acad Sci U S A. 1993;90:7966-9.

7. Dunlop BW, Nemeroff CB. The role of dopamine in the pathophysiology of depression. Arch Gen Psychiatry. 2007;64:327-37.

8. Martinot M, Bragulat V, Artiges E, Dolle F, Hinnen F, Jouvent R, et al. Decreased presynaptic dopamine function in the left caudate of depressed patients with affective flattening and psychomotor retardation. Am J Psychiatry. 2001;158:314-6.

9. Camardese G, De Risio L, Di Nicola M, Pucci L, Cocciolillo F, Bria P, et al. Changes of dopamine transporter availability in depressed patients with and without anhedonia: a 123I-N-omega-fluoropropyl-carbomethoxy-3beta- (4-lodophenyl) tropane SPECT study. Neuropsychobiology. 2014;70:235-43.

10. Tye KM, Mirzabekov J, Warden MR, Ferenczi EA, Tsai HC, Finkelstein J, et al. Dopamine neurons modulate neural encoding and expression of depressionrelated behaviour. Nature. 2013:493:537-41.

11. Balcita-Pedicino JJ, Omelchenko N, Bell R, Sesack SR. The inhibitory influence of the lateral habenula on midbrain dopamine cells: ultrastructural evidence for indirect mediation via the rostromedial mesopontine tegmental nucleus. J Comp Neurol. 2011;519:1143-64.

12. Jhou TC, Fields HL, Baxter MG, Saper CB, Holland PC. The rostromedial tegmental nucleus (RMTg), a GABAergic afferent to midbrain dopamine neurons, encodes aversive stimuli and inhibits motor responses. Neuron. 2009;61:786-800.

13. Kaufling J, Veinante P, Pawlowski SA, Freund-Mercier MJ, Barrot M. Afferents to the GABAergic tail of the ventral tegmental area in the rat. J Comp Neurol. 2009;513:597-621.

14. Stamatakis AM, Stuber GD. Activation of lateral habenula inputs to the ventral midbrain promotes behavioral avoidance. Nat Neurosci. 2012;15:1105-7.

15. Jhou TC, Geisler S, Marinelli M, Degarmo BA, Zahm DS. The mesopontine rostromedial tegmental nucleus: A structure targeted by the lateral habenula that projects to the ventral tegmental area of Tsai and substantia nigra compacta. J Comp Neurol. 2009;513:566-96.

16. Di Chiara G, Imperato A. Drugs abused by humans preferentially increase synaptic dopamine concentrations in the mesolimbic system of freely moving rats. Proc Natl Acad Sci USA 1988;85:5274-8.

17. Hong S, Jhou TC, Smith M, Saleem KS, Hikosaka O. Negative reward signals from the lateral habenula to dopamine neurons are mediated by rostromedial tegmental nucleus in primates. J Neurosci. 2011;31:11457-71.

18. Lecca $S$, Melis $M$, Luchicchi $A$, Muntoni AL, Pistis $M$. Inhibitory inputs from rostromedial tegmental neurons regulate spontaneous activity of midbrain dopamine cells and their responses to drugs of abuse. Neuropsychopharmacology. 2012;37:1164-76.

19. Barrot M, Sesack SR, Georges F, Pistis M, Hong S, Jhou TC. Braking dopamine systems: a new GABA master structure for mesolimbic and nigrostriatal functions. J Neurosci. 2012;32:14094-101.

20. Fakhoury $M$. The tail of the ventral tegmental area in behavioral processes and in the effect of psychostimulants and drugs of abuse. Prog Neuropsychopharmacol Biol Psychiatry. 2018;84:30-38.

21. Jhou TC, Good CH, Rowley CS, Xu SP, Wang H, Burnham NW, et al. Cocaine drives aversive conditioning via delayed activation of dopamine-responsive habenular and midbrain pathways. J Neurosci. 2013;33:7501-12.

22. Matsui A, Jarvie BC, Robinson BG, Hentges ST, Williams JT. Separate GABA afferents to dopamine neurons mediate acute action of opioids, development of tolerance, and expression of withdrawal. Neuron. 2014;82:1346-56.

23. Jhou TC, Xu SP, Lee MR, Gallen $C L$, Ikemoto S. Mapping of reinforcing and analgesic effects of the mu opioid agonist endomorphin-1 in the ventral midbrain of the rat. Psychopharmacology (Berl). 2012;224:303-12.

24. Glover EJ, McDougle MJ, Siegel GS, Jhou TC, Chandler LJ. Role for the rostromedial tegmental nucleus in signaling the aversive properties of alcohol. Alcohol Clin Exp Res. 2016;40:1651-61.

25. Fu R, Chen X, Zuo W, Li J, Kang S, Zhou LH, et al. Ablation of mu opioid receptorexpressing GABA neurons in rostromedial tegmental nucleus increases ethanol consumption and regulates ethanol-related behaviors. Neuropharmacology. 2016;107:58-67.
26. Sheth C, Furlong TM, Keefe KA, Taha SA. Lesion of the rostromedial tegmental nucleus increases voluntary ethanol consumption and accelerates extinction of ethanol-induced conditioned taste aversion. Psychopharmacology (Berl). 2016;233:3737-49.

27. Cui Y, Yang Y, Ni Z, Dong Y, Cai G, Foncelle A, et al. Astroglial Kir4.1 in the lateral habenula drives neuronal bursts in depression. Nature. 2018;554:323-27.

28. Li K, Zhou T, Liao L, Yang Z, Wong C, Henn F, et al. betaCaMKII in lateral habenula mediates core symptoms of depression. Science. 2013;341:1016-20.

29. Lecca S, Pelosi A, Tchenio A, Moutkine I, Lujan R, Herve D, et al. Rescue of GABAB and GIRK function in the lateral habenula by protein phosphatase $2 A$ inhibition ameliorates depression-like phenotypes in mice. Nat Med. 2016;22:254-61.

30. Barrot $M$, Thome J. Discovering a new anatomical structure in the brain: implications for neuropsychiatry and therapy. World J Biol Psychiatry. 2011;12:19-22.

31. Farrell MS, Roth BL. Pharmacosynthetics: Reimagining the pharmacogenetic approach. Brain Res. 2013;1511:6-20.

32. Li J, Kang S, Fu R, Wu L, Wu W, Liu H, et al. Inhibition of AMPA receptor and CaMKII activity in the lateral habenula reduces depressive-like behavior and alcohol intake in rats. Neuropharmacology. 2017;126:108-20.

33. Li J, Bian W, Dave V, Ye JH. Blockade of GABA(A) receptors in the paraventricular nucleus of the hypothalamus attenuates voluntary ethanol intake and activates the hypothalamic-pituitary-adrenocortical axis. Addict Biol. 2011;16:600-14.

34. Simms JA, Steensland $P$, Medina $B$, Abernathy KE, Chandler $L$, Wise $R$, et al. Intermittent access to $20 \%$ ethanol induces high ethanol consumption in LongEvans and Wistar rats. Alcohol Clin Exp Res. 2008;32:1816-23.

35. Wise RA. Voluntary ethanol intake in rats following exposure to ethanol on various schedules. Psychopharmacologia. 1973;29:203-10.

36. Fu R, Mei Q, Zuo W, Li J, Gregor D, Bekker A, et al. Low-dose ethanol excites lateral habenula neurons projecting to VTA, RMTg, and raphe. Int J Physiol Pathophysiol Pharmacol. 2017;9:217-30.

37. Fu R, Zuo W, Gregor D, Li J, Grech D, Ye JH. Pharmacological manipulation of the rostromedial tegmental nucleus changes voluntary and operant ethanol selfadministration in rats. Alcohol Clin Exp Res. 2016;40:572-82.

38. Zuo W, Fu R, Hopf FW, Xie G, Krnjevic K, Li J, et al. Ethanol drives aversive conditioning through dopamine 1 receptor and glutamate receptor-mediated activation of lateral habenula neurons. Addict Biol. 2017;22:103-16.

39. Norris C, Loureiro M, Kramar C, Zunder J, Renard J, Rushlow W, et al. Cannabidiol Modulates Fear Memory Formation Through Interactions with Serotonergic Transmission in the Mesolimbic System. Neuropsychopharmacology. 2016;41:2839-50.

40. Wang N, Ge F, Cui C, Li Y, Sun X, Sun L, et al. Role of glutamatergic projections from the ventral $C A 1$ to infralimbic cortex in context-induced reinstatement of heroin seeking. Neuropsychopharmacology. 2018;43:1373-84.

41. Li J, Fu C, Liu H, Fu R, Zuo W, Kang S, et al. Electroacupuncture attenuates hyperalgesia in rats withdrawn from chronic alcohol drinking via habenular $\mathrm{Mu}$ opioid receptors. Alcohol Clin Exp Res. 2017;41:637-43.

42. Nestler EJ, Carlezon WA Jr. The mesolimbic dopamine reward circuit in depression. Biol Psychiatry. 2006;59:1151-9.

43. Goncalves L, Sego C, Metzger M. Differential projections from the lateral habenula to the rostromedial tegmental nucleus and ventral tegmental area in the rat. J Comp Neurol. 2012;520:1278-300.

44. Lammel S, Lim BK, Ran C, Huang KW, Betley MJ, Tye KM, et al. Input-specific control of reward and aversion in the ventral tegmental area. Nature. 2012;491:212-7.

45. Fadda F, Rossetti ZL. Chronic ethanol consumption: from neuroadaptation to neurodegeneration. Prog Neurobiol. 1998;56:385-431.

46. Kang $\mathrm{S}, \mathrm{Li} \mathrm{J}$, Bekker $\mathrm{A}, \mathrm{Ye} \mathrm{JH}$. Rescue of glutamate transport in the lateral habenula alleviates depression- and anxiety-like behaviors in ethanol-withdrawn rats. Neuropharmacology. 2018;129:47-56.

47. Madden JS. Alcohol and depression. Br J Hosp Med. 1993;50:261-4.

48. Heinz A, Schmidt LG, Reischies FM. Anhedonia in schizophrenic, depressed, or alcohol-dependent patients-neurobiological correlates. Pharmacopsychiatry. 1994;27:7-10.

49. Roni MA, Rahman S. Lobeline attenuates ethanol abstinence-induced depressionlike behavior in mice. Alcohol. 2017;61:63-70.

50. Getachew B, Hauser SR, Taylor RE, Tizabi Y. Alcohol-induced depressive-like behavior is associated with cortical norepinephrine reduction. Pharmacol Biochem Behav, 2010;96:395-401.

51. Glover EJ, Starr EM, Chao Y, Burnham NW, Jhou TC, Chandler LJ. Inhibition of the rostromedial tegmental nucleus reverses withdrawal-induced negative affect. Alcohol Clin Exp Res. 2017;41:118A.

52. Kaufling J, Aston-Jones G. Persistent adaptations in afferents to ventral tegmental dopamine neurons after opiate withdrawal. J Neurosci. 2015;35:10290-303.

53. Burnett EJ, Chandler LJ, Trantham-Davidson H. Glutamatergic plasticity and alcohol dependence-induced alterations in reward, affect and cognition. Prog Neuropsychopharmacol Biol Psychiatry. 2016;65:309-20. 
54. Brinschwitz K, Dittgen A, Madai VI, Lommel R, Geisler S, Veh RW. Glutamatergic axons from the lateral habenula mainly terminate on GABAergic neurons of the ventral midbrain. Neuroscience. 2010;168:463-76.

55. Kang S, Li J, Zuo W, Fu R, Gregor D, Krnjevic K, et al. Ethanol withdrawal drives anxiety-related behaviors by reducing M-type potassium channel activity in the lateral habenula. Neuropsychopharmacology. 2017;42:1813-24.

56. Brodie MS, Shefner SA, Dunwiddie TV. Ethanol increases the firing rate of dopamine neurons of the rat ventral tegmental area in vitro. Brain Res. 1990;508:65-9.

57. Gessa GL, Muntoni F, Collu M, Vargiu L, Mereu G. Low doses of ethanol activate dopaminergic neurons in the ventral tegmental area. Brain Res. 1985;348:201-3.

58. Imperato A, Di Chiara G. Preferential stimulation of dopamine release in the nucleus accumbens of freely moving rats by ethanol. J Pharmacol Exp Ther. 1986;239:219-28.

59. Bailey CP, Manley SJ, Watson WP, Wonnacott S, Molleman A, Little HJ. Chronic ethanol administration alters activity in ventral tegmental area neurons after cessation of withdrawal hyperexcitability. Brain Res. 1998;803:144-52.

60. Matsumoto $M$, Hikosaka $O$. Lateral habenula as a source of negative reward signals in dopamine neurons. Nature. 2007;447:1111-U11.

61. Tepper JM, Lee CR. GABAergic control of substantia nigra dopaminergic neurons. Prog Brain Res. 2007;160:189-208.

62. File SE, Baldwin HA. Effects of beta-carbolines in animal models of anxiety. Brain Res Bull. 1987;19:293-9.
63. Sanchez-Catalan MJ, Faivre F, Yalcin I, Muller MA, Massotte D, Majchrzak M, et al Response of the tail of the ventral tegmental area to aversive stimuli. Neuropsychopharmacology. 2017;42:638-48.

64. Markou A, Koob GF. Postcocaine anhedonia. An animal model of cocaine withdrawal. Neuropsychopharmacology. 1991;4:17-26.

65. Kaufling J, Veinante P, Pawlowski SA, Freund-Mercier MJ, Barrot M. Gammaaminobutyric acid cells with cocaine-induced DeltaFosB in the ventral tegmental area innervate mesolimbic neurons. Biol Psychiatry. 2010;67:88-92.

66. McLaughlin I, Dani JA, De Biasi M. The medial habenula and interpeduncular nucleus circuitry is critical in addiction, anxiety, and mood regulation. J Neurochem. 2017;142:130-43.

67. Berton O, Hahn CG, Thase ME. Are we getting closer to valid translational models for major depression? Science. 2012;338:75-9.

68. Koob GF, Le Moal M. Drug addiction, dysregulation of reward, and allostasis. Neuropsychopharmacology. 2001;24:97-129.

69. Volkow ND, Fowler JS, Wang GJ, Goldstein RZ. Role of dopamine, the frontal cortex and memory circuits in drug addiction: insight from imaging studies. Neurobiol Learn Mem. 2002;78:610-24.

70. Verendeev A, Riley AL. The role of the aversive effects of drugs in self-administration assessing the balance of reward and aversion in drug-taking behavior. Behav Pharmacol. 2013;24:363-74.

71. Paxinos G, Watson C (2007) The rat brain in stereotaxic coordinates. 6th Edition, Academic Press, San Diego. 